\title{
Anabases
}

ANABASES Traditions et réceptions de l'Antiquité

1 | 2005

Varia

\section{Historicisation, déshistoricisation : relire Ernesto De Martino}

Pierre Cordier

\section{(2) OpenEdition}

1 Journals

Édition électronique

URL : http://journals.openedition.org/anabases/1502

DOI : 10.4000/anabases. 1502

ISSN : 2256-9421

Éditeur

E.R.A.S.M.E.

\section{Édition imprimée}

Date de publication : 1 mars 2005

Pagination : 288-292

ISSN : 1774-4296

\section{Référence électronique}

Pierre Cordier, «Historicisation, déshistoricisation : relire Ernesto De Martino », Anabases [En ligne], 1] 2005, mis en ligne le 01 octobre 2011, consulté le 21 octobre 2019. URL : http:// journals.openedition.org/anabases/1502 ; DOI : 10.4000/anabases.1502

Ce document a été généré automatiquement le 21 octobre 2019

(c) Anabases 


\title{
Historicisation, déshistoricisation : relire Ernesto De Martino
}

\author{
Pierre Cordier
}

1 Les uniformes disciplinaires de l'Université n'étaient pas coupés pour Ernesto De Martino (1908-1965), qui se définissait lui-même comme un «intellettuale di transizione, lacerato dalle contradizioni $\Perp^{58}$. Philosophe, historien des religions, ethnologue, De Martino ne se laissa pas davantage enfermer dans un unique territoire chronologique. Sa réflexion supposait un incessant va-et-vient entre le présent et le passé le plus lointain : son ethnologie passait par l'histoire. Consacrer le premier Atelier à ce penseur paradoxal s'imposait à plus d'un titre : pluridisciplinaire, comparatiste, incessamment nourrie de références antiques, l'oeuvre de De Martino suscite, depuis une quinzaine d'années, un intérêt croissant, marqué par la réédition en Italie de quelques-uns de ses écrits majeurs, accompagnés d'introductions épistémologiques ${ }^{59}$. Le rôle joué par De Martino au sein des réseaux scientifiques et éditoriaux contemporains est bien mis en évidence par la publication de quelques volets de sa correspondance ${ }^{60}$.

2 La renaissance de De Martino déborde la péninsule italienne : bien que la France n'ait guère prêté attention à cet auteur que dans les années $1960^{61}$, une série d'articles et un dossier parus dans la revue Gradhiva $(26,1999)$ montrent que l'ethnologue italien acquiert progressivement le statut d'auteur classique de l'anthropologie religieuse ${ }^{62}$. En témoigne également la réédition de ses traductions en français ${ }^{63}$. Le volume du Monde magique est accompagné d'une riche postface de Silvia Mancini, que l'on complètera par la lecture du compte rendu de l'ouvrage dans la revue Gradhiva ${ }^{64}$. Cette activité éditoriale, jointe à la publication d'une série d'études ou d'articles sur Ernesto De Martino, invite à redécouvrir une pensée souvent connue de manière indirecte et biaisée.

3 La genèse de la méthode et des thèses demartiniennes s'inscrit dans le contexte d'une formation universitaire et d'un réseau d'échanges intellectuels éclectiques. A l'origine de l'intérêt que l'ethnologue témoigne à l'Antiquité, qui lui fournit d'innombrables motifs d'analogie, deux études soulignent l'influence de Vittorio Macchioro, l'auteur du 
Zagreus, un ouvrage sur l'orphisme publié pour la première fois à Bari, en 1920, et d'Adolfo Omodeo, historien du christianisme antique ${ }^{65}$. Même si, très tôt, De Martino prend ses distances avec l'idée d'une continuité linéaire et d'une filiation des modalités de l'expérience religieuse orphique et de celles du christianisme paulinien, les héritages culturels de l'Antiquité lui fournissent un instrument d'analyse : l'idée de syncrétisme ${ }^{66}$. La question des manifestations magico-religieuses dans le Mezzogiorno le confronte brutalement à une autre question, celle des vestiges (relitti) des attitudes culturelles et de leur permanence au présent. D'une certaine façon, la conversion de De Martino à l'ethnologie de terrain surgit d'une expérience de l'Altérité : l'antique semble faire irruption dans le contemporain; l'existence, au sein du présent, d'enclaves primitives révèle la pluralité des temps sociaux vécus au sein d'une même culture. De Martino repère ces survivances dans le rituel des lamentations funèbres, dans le mauvais oeil, dans le tarentisme ${ }^{67}$. La compréhension d'un présent étrange et hétérogène impose une enquête rigoureuse sur les origines: pour comprendre les séquences gestuelles du tarentisme méridional, l'ethnologue enquête dans l'Antiquité grecque sur les représentations de la piqûre d'insecte (oistro1) ou les usages rituels de la balançoire (aijwvrhsii).

4 Le parti-pris d'une ethnologie métropolitaine procède du vertige ressenti devant l'altérité des faits religieux méridionaux : l'enquête sur les Lucaniens revêt un caractère dialectique et aboutit à un examen critique des présupposés et des convictions de l'observateur, qui est la condition et le mode opératoire du regard ethnologique ${ }^{68}$. L'ethnologue des faits métropolitains fait l'expérience de l'autre au sein du soi, choc fondamental pour la définition de cette position dialectique, entre passé et présent, entre sujet et objet, entre empirique et théorique, entre connaissance et action, qui caractérise l'« ethnocentrisme critique ${ }^{69}$. Aussi l'enquête sur les antécédents, tout en refusant la tentation du naturalisme, rompt-elle avec l'historicisme de Croce, et ne se satisfait-elle pas, à titre d'explication, d'un simple inventaire des origines. L'archéologie, au sens thucydidéen du terme, est chez Ernesto De Martino à l'opposé d'une Quellenforschung historiciste : le passé n'a ni plus de substance ni plus d'autorité pour le présent. A lui seul, il n'explique rien. Une enquête comparative doit chercher les principes de l'éternel retour ou de la rémanence entêtée qui caractérise de certaines constructions sociales, tels le mythe et le rite ${ }^{70}$. Dès la seconde édition de Morte e pianto rituale nel mondo antico: dal lamento pagano al pianto di Maria, paru en 1958, le titre remplace la référence au paganisme par une qualification purement chronologique, comme pour souligner qu'il s'agit moins d'une survivance, de la projection d'un fragment de système cloisonné dans un autre, que d'une analogie fonctionnelle ${ }^{71}$. L'intérêt de De Martino pour la continuité historique des pratiques, au sein d'une même nébuleuse culturelle, et pour les comparaisons, d'un système culturel à l'autre, reflète en outre son engagement politique et l'influence du communisme ${ }^{72}$ : l'écrasement, la souffrance, la dégradation du dominé produisent des réponses récurrentes, voire des manifestations tendant vers l'universel, auxquelles l'ethnologue s'attache comme à des embryons étouffés de résistance, comme si la crise du sujet était une modalité, personnelle et intime, d'une la lutte des classes qui traverse l'Histoire ${ }^{73}$. Bien qu'elle soit étroitement liée à des variables historiques, la crise, dans sa forme, tend à présenter un profil constant ${ }^{74}$.

5 Ainsi, communes aux expériences religieuses de l'antiquité et des Lucaniens qu'il observe, les "techniques de l'esprit» - la transe, la fascination magique et les techniques hypnogènes - sont-elles à la fois les symptômes et les réponses à des 
situations de crise individuelle, telles que le deuil et la souffrance psychologique; la ritualisation des comportements autorise une forme de dédoublement du sujet, qui se trouve "déshistoricisé ", c'est-à-dire coupé de son monde culturel et historique commun. En réponse à cette rupture du lien et du temps sociaux, le rituel prend en charge, de manière institutionnelle, la crise individuelle et assure la reconstitution progressive de la personne. Le rituel est un drame, à la fois au sens de situation critique et comme représentation: d'une certaine façon, le sujet mime rituellement une forme pathologique de désocialisation, en faisant alterner des phases de prostration et des phases d'exubérance rituelle ${ }^{75}$. À l'échelle collective, les sociétés connaissent, avec leurs hantises de la chute et leurs mises en scènes d'apocalypses, des phénomènes analogues $^{76}$. Les derniers textes de De Martino s'efforcent de traduire et de représenter l'absence à soi, l'« écroulement de la présence » à l'échelle de groupes sociaux élargis.

6 Contrairement à Louis Gernet, De Martino n'esquive pas la question de la crise psychologique associée aux paroxysmes rituels et ne fait pas disparaitre l'expérience individuelle en l'escamotant sous une règle ou un faisceau de représentations sociales ${ }^{77}$. Le regard ethnologique, chez De Martino, se concentre sur l'individu, avec son histoire propre et ses accidents de parcours, pour saisir les symptômes de la désorganisation du sujet et dresser le diagnostic clinique d'une crise de la socialisation. Bien qu'elle s'attache à l'infiniment particulier et qu'elle puisse, à bien des égards, apparaître comme une pensée du kairos, la réflexion de De Martino n'oppose pas radicalement l'individuel au collectif: de rite en rite, la société sait réparer ce qu'elle détruit; il y a une réponse sociale à la souffrance sociale. En ce sens, De Martino apparaît comme l'un des pères fondateurs de l'ethnopsychiatrie: la question de l'efficacité symbolique des rituels est au centre de son enquête et l'ethnologue l'affronte sans éluder le problème ; loin de déboucher sur une rupture méthodologique, un saut dans l'irrationnel et le recours à une dimension sacrée transcendante ou, au moins, hors de portée de l'analyste des faits sociaux, l'efficacité symbolique est interprétée comme un signe de la capacité «naturante » de la culture; d'une certaine façon, la Nature, chez De Martino, ne fait jamais directement l'objet de l'expérience humaine. La culture, seul environnement humain, est un phénomène total, au point que le symbolique sait $s^{\prime} i m p o s e r$ par-dessus les évidences physiques ${ }^{78}$. Cette définition radicale de la culture, qu'il est aisé de caricaturer, est à l'origine de la longue quarantaine à laquelle l'auteur du Monde magique a été soumis. Il serait réducteur de cantonner l'usage de De Martino à la sphère du "magico-religieux ». Par-delà l'intérêt de l'ethnologue pour l'irrationnel, voire l'occulte, à la croisée de la philosophie de l'histoire, de l'enquête ethnologique et de l'histoire culturelle, cette conception rayonnante de la culture manifeste le refus de réduire le rapport que les autres entretiennent avec le sacré à une simple question de croyance et rappelle que, même si l'histoire y ménage des points de fuite, des tourbillons et des turbulences, la culture est vécue comme un absolu. 


\section{NOTES}

58. Expression citée dans l'introduction de Compagni e amici. Lettere di Ernesto De Martina e Pietro Secchia, a cura di R. DI DONATO, Florence, La Nuova Italia, 1993, p. XXI et n. 7.

59. Outre Naturalismo e storicismo nell'etnologia, intr. (p. 9-49) a cura di S. DE MATTEIS, Lecce, Argo, 1997, on renverra à une bibliographie mise à jour jusqu'à 1998 in R. DI DONATO, I Greci selvaggi. Antropologia storica di Ernesto De Martino, Rome, Manifestolibri, 1999, p. 211-228.

60. Notamment La collana viola. Lettere 1945-1950 (Cesare Pavese, Ernesto de Martino), a cura di P. ANGELINI, Turin, Bollati Boringhieri (Temi, 20), 1991.

61. D. FABRE, “ Un rendez-vous manqué : Ernesto De Martino et sa réception en France ", L'Homme 151 (1999), p. 207-236 et id., “De Martino altrove : sulla sua ricezione francese ”, in Ernesto De Martino nella cultura europea, a cura di C. GALLINI e M. MASSENZIO (Anthropos, 32), 1997, p. 139-176.

62. C. BERGÉ, “Lectures de De Martino en France aujourd'hui ”, Ethnologie française 31 (2001), p. 537-547.

63. L'œuvre de Ernesto De Martino, I, Le monde magique (tr. fr. par M. Baudoux, 1971, postface de S. Mancini p. 285-575), II, Italie du Sud et magie (tr. fr. par C. Poncet, 1963), III, La terre du remords (tr. fr. par C. Poncet, 1966), Le Plessis-Robinson, Institut Synthélabo pour le progrès de la connaissance (Les empêcheurs de penser en rond), 1999, 3 vol.

64. D. DUBUisson, “L'anthropologie au risque de la métaphysique ”, Gradhiva 28 (2000), p. 114-117.

65. L. REBAUDO, "Vittorio Macchioro, storico e archeologo. Gli scritti", in R. DI DONATO, La contraddizione felice? Ernesto De Martino e gli altri, Pise, ETS (Universitas, n. s., 17 ; Scienze dell'uomo e della società, 2) 1990, p. 205-220 et P. CERCHI, Il signore del limite. Tre variazione critiche su Ernesto De Martino, Naples, Liguori, (Anthropos, 27), 1994, p. 9-15 ; S. MANCINI, “ Mimétisme et rite : de la lamentation funéraire à la phénoménologie de Padre Pio", Revue de l'histoire des religions 221/3 (2004), p. 327-353.

66. R. DI DONATO, I Greci selvaggi, notamment le chapitre “ sincretismi ", p. 153-168.

67. Pour un état des recherches sur le tarentisme après De Martino, cf. A. TURCHINI, Morso, morbo, morte. La tarantola fra cultura medica e terapia popolare, Milan, Franco Angeli, 1987, p. 15-46, le chapitre "Terra del rimorso, 20 anni dopo".

68. C. GALLINI, “Note su De Martino e l'etnocentrismo critico", Orientamenti marxisti e studi antropologici italiani, Milan, Franco Angeli (Quaderni di “Problemi del Socialismo"), 1980, p. 211-222 ; P. CERCHI, Il peso dell'ombra : l'etnocentrismo critico di Ernesto De Martino e il problema dell'autocoscienza culturale, Naples, Liguori (Anthropos, 30), 1996.

69. S. MANCINI, "La notion d'ethnocentrisme critique et l'anthropologie contemporaine", Gradhiva 22 (1997), p. 1-14, et M. MASSENZIO, Sacré et identité ethnique. Frontières et ordre du monde, préface de M. Augé, traduction de Federica Giardini et Valeria Giardini révisée par Giordana Charuty, Paris, Editions de l'EHESS, 1999 (tr. de Sacro e identità etnica. Senso del mondo e linea di confine, Milan, Franco Angeli, 1994), p. 38-55.

70. M. MASSENZIO, “La ripetizione della ripetizione", Ernesto De Martino nella cultura europea, p. 237-246 ; L. LATTARULO, Esistenza e valore: Croce, De Martino e la crisi dello storicismo italiano, Rome, Cadmo (Biblioteca di scienze filosofiche e sociali; Centro Mario Rossi per gli studi filosofici dell'Università di Siena, 2), 1987.

71. Morte e pianto rituale. Dal lamento funebre antico al pianto di Maria, introduzione di C. Gallini, Turin, Bollati Boringhieri 1975 (réimpr. 2000).

72. Cf. R. DI DonATO, Compagni e amici : lettere di Ernesto De Martino e Pietro Secchia, Scandicci, La Nuova Italia (Biblioteca di Storia, 44), 1993 pour l'engagement de De Martino et, pour le lien entre 
son enquête dans le Mezzogiorno et son engagement politique, Giordana Charuty, “L'ethnologue et le citoyen ", Gradhiva 26 (1999), p. 83-98.

73. C.A. AUGIERI, La letteratura e le forme dell'oltrepassamento : Bachtin, De Martino, Jakobson, Lotman, Lecce, Manni, 2002.

74. C GALLINI, "Ripensando l'autonomia relativa del simbolico", La contraddizione felice?, p. 129-142.

75. P. CERCHI, M. CERCHI, Ernesto De Martino. Dalla crisi della presenza alla comunità umana, Naples, Liguori, 1987.

76. A. momigliano, "Per la Storia delle religioni in Italia contemporanea, Antonio Banfi ed Ernesto De Martino tra persona e apocalissi ", in La contraddizione felice, p. 13-36 ; G. SASso, "Le apocalissi culturali. Ultime riflessioni di Ernesto De Martino ", Nuovi Argomengi, 5 (gennaio-marzo 1999), p. 35-80 ; C. SEVERI, “Une pensée inachevée. L'utopie anthropologique de Ernesto De Martino ”, Gradhiva 26 (1999), p. 99-107; M. MASSENZIO, “Le volume-testament d'Ernesto De Martino ", ibid., p. 53-64.

77. J.-P. VERNANT, “Persona e biografia : un intervento ”, in La contraddizione felice ?, p. 37-39.

78. P. CERCHI, “La nozione di cultura e la realtà dei poteri magici ”, in Ernesto De Martino nella cultura europea, p. 259-268.

\section{AUTEUR}

PIERRE CORDIER

pierre.cordier@free.fr 